\title{
Parametrization of the driven betatron oscillation
}

\author{
R. Miyamoto and S. E. Kopp \\ Department of Physics, University of Texas at Austin, Austin, Texas 78712, USA
}

A. Jansson and M. J. Syphers

Fermi National Accelerator Laboratory, Batavia, Illinois 60510, USA

(Received 18 September 2007; published 27 August 2008)

\begin{abstract}
An AC dipole is a magnet which produces a sinusoidally oscillating dipole field and excites coherent transverse beam oscillations in a synchrotron. By observing this driven coherent oscillation, the linear optical parameters can be directly measured at locations of the beam position monitors. The driven oscillations induced by an AC dipole will generate a phase space ellipse which differs from that of free oscillations. If not properly accounted for, this difference can lead to misinterpretations of the actual optical parameters, for instance, $6 \%$ or more in the cases of the Tevatron, RHIC, or LHC. This paper shows that the effect of an AC dipole on the observed linear optics is identical to that of a thin lens quadrupole. By introducing a new amplitude function to describe this new phase space ellipse, the motion produced by an $\mathrm{AC}$ dipole becomes easier to interpret. The introduction of this new amplitude function also helps measurements of the normal Courant-Snyder parameters based on beam position data taken under the influence of an $\mathrm{AC}$ dipole. This new parametrization of driven oscillations is presented and is used to interpret data taken in the FNAL Tevatron using an AC dipole.
\end{abstract}

DOI: 10.1103/PhysRevSTAB.11.084002

PACS numbers: 41.85. $-\mathrm{p}, 29.27 .-\mathrm{a}$

\section{INTRODUCTION}

In a modern accelerator, transverse motion of charged particles is stabilized with linear forces of quadrupole magnets and, as a result, the particles undergo oscillations around the ideal trajectory, called betatron oscillations [1]. If observed at one location in an accelerator, the oscillations are discrete. The betatron tune [2] is the frequency of such discrete oscillations in units of the revolution frequency. The amplitude of the betatron oscillations at a location in an accelerator is determined by a parameter called $\beta$ function. The relative phase difference of the betatron oscillations between two locations in an accelerator is called betatron phase advance. If quadrupole magnets in an accelerator have (gradient) errors, the $\beta$ function and betatron phase advance become different from their design values and performance of the accelerator can be degraded. One purpose of an $\mathrm{AC}$ dipole is to measure the $\beta$ function and betatron phase advance and to find such errors. Individual particles within the beam oscillate incoherently and we can only observe motion of the beam centroid with a beam position monitor (BPM). Hence, to observe the betatron oscillations and measure parameters such as the $\beta$ function or the betatron phase advance, coherent oscillations must be excited (Fig. 1). An AC dipole is a tool to excite coherent oscillations of the beam [3], much like a kicker/pinger magnet, but over a longer time period.

An AC dipole excites coherent transverse oscillations with a sinusoidally oscillating dipole field. It drives the beam close to the betatron frequency, typically, for several thousands of revolutions. If the amplitude of its oscillating magnetic field is adiabatically ramped up and down, it can produce large coherent oscillations without decoherence or emittance growth [3]. This property makes it a useful diagnostic tool of a synchrotron. AC dipoles have been employed in the BNL AGS and RHIC [3-5], CERN SPS [6,7], and FNAL Tevatron [8-10]. There is an ongoing project to develop AC dipoles for LHC as well [11].

When the beam is driven by an AC dipole, its motion is governed by two driving terms and the influence of the

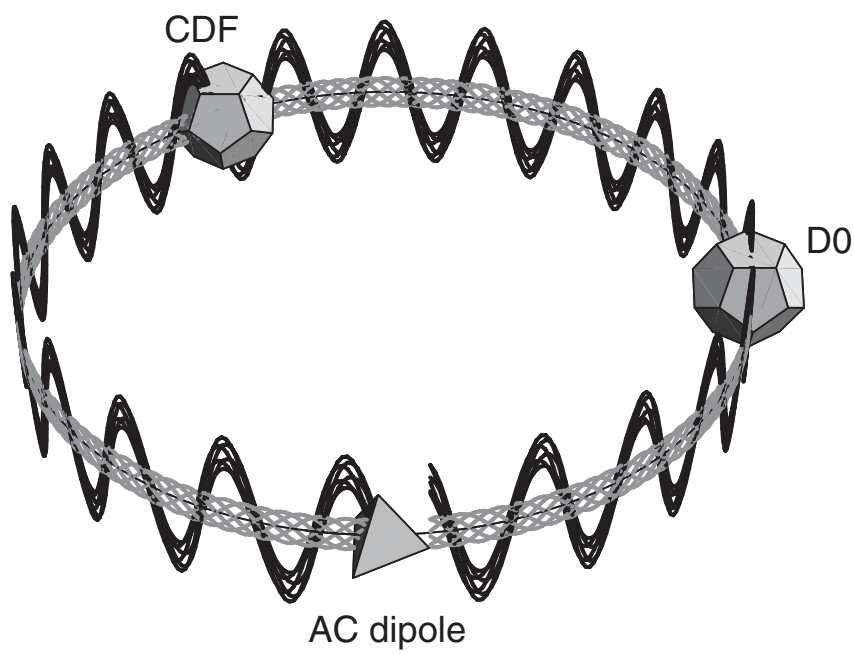

FIG. 1. Schematic of incoherent free oscillations (gray) and excited coherent oscillations (black) of charged particles in the Tevatron. Because individual particles within the beam oscillate incoherently, coherent oscillations must be excited to observe the betatron oscillations and measure parameters such as the $\beta$ function. An AC dipole is one of such tools to excite coherent oscillations of the beam. 
lesser driving term makes driven oscillations different from the free oscillations. Although this difference has typically been ignored in previous analyses [5,12], it could affect interpretation of the $\beta$ function more than $12 \%$ in a typical operational condition of the Tevatron.

This paper proceeds as follows. Section II discusses the two driving terms produced by an AC dipole and presents a new formulation of driven oscillations which is suited to simultaneously treat the two driving terms. Section III discusses the difference between free and driven oscillations and the influence of this difference on measurements of the $\beta$ function, based on an analogy between the driven motion and motion under the influence of a thin gradient error [13]. Section IV presents a few examples of the difference between free and driven oscillations observed in the Tevatron and explains how to measure the $\beta$ function by appropriate analyses of data of driven oscillations.

\section{A MODEL OF DRIVEN OSCILLATIONS}

\section{A. Two driving terms of an oscillating dipole field}

The tune of an AC dipole $\nu_{\text {acd }}$ is defined as the ratio between the frequencies of the AC dipole $f_{\text {acd }}$ and the beam revolution $f_{\text {rev }}: \nu_{\text {acd }} \equiv f_{\text {acd }} / f_{\text {rev }}$. In the following, for any tunes, only their fractional parts are considered. For instance, if $f_{\text {acd }} / f_{\text {rev }}$ is larger than one, $\nu_{\text {acd }}$ means the fractional part of $f_{\text {acd }} / f_{\text {rev }}$. Because the beam sees an AC dipole only once in a revolution, the beam is driven by a pair of driving terms with driving tunes $\nu_{\text {acd }}$ and $1-\nu_{\text {acd }}$ (cf. Nyquist sampling theorem) [14]. Obviously, the driving term closer to the machine tune $\nu$ has a bigger influence on a particle. Hence, in the following, the driving term closer to $\nu$ is called the primary and the other is called the secondary. A symbol $\nu_{d}$ is used for the primary driving tune (Fig. 2):

$$
\nu_{d} \equiv \begin{cases}\nu_{\text {acd }} & \text { when }\left|\nu_{\text {acd }}-\nu\right|<\left|\left(1-\nu_{\text {acd }}\right)-\nu\right| \\ 1-\nu_{\text {acd }} & \text { when }\left|\left(1-\nu_{\text {acd }}\right)-\nu\right|<\left|\nu_{\text {acd }}-\nu\right|\end{cases}
$$

For example, the frequencies of the AC dipole and beam revolution in the Tevatron are $f_{\text {acd }} \simeq 20.5 \mathrm{kHz}$ and $f_{\text {rev }} \simeq$ $47.7 \mathrm{kHz}$ and, hence, the tune of the AC dipole is $\nu_{\text {acd }}=$ $20.5 / 47.7 \simeq 0.43$. Because the machine tune of the Tevatron is $\nu \simeq 0.58,1-\nu_{\text {acd }} \simeq 0.57$ is the primary driving tune and $\nu_{\text {acd }} \simeq 0.43$ is secondary. We note that machine tunes near 0.5 tend to exaggerate the influence of the secondary driving term.

The difference between the primary driving tune and the machine tune, $\delta_{d} \equiv \nu_{d}-\nu$, is an important parameter of driven oscillations. As $\delta_{d} \rightarrow 0$, the influence of the primary driving term becomes dominant and the secondary driving term can be ignored. However, a finite tune spread of the beam can cause beam losses if $\left|\delta_{d}\right|$ is too small (Fig. 2). In the Tevatron, without a special tune-up, the limit of $\left|\delta_{d}\right|$ is about $0.005-0.015$ to prevent beam losses.

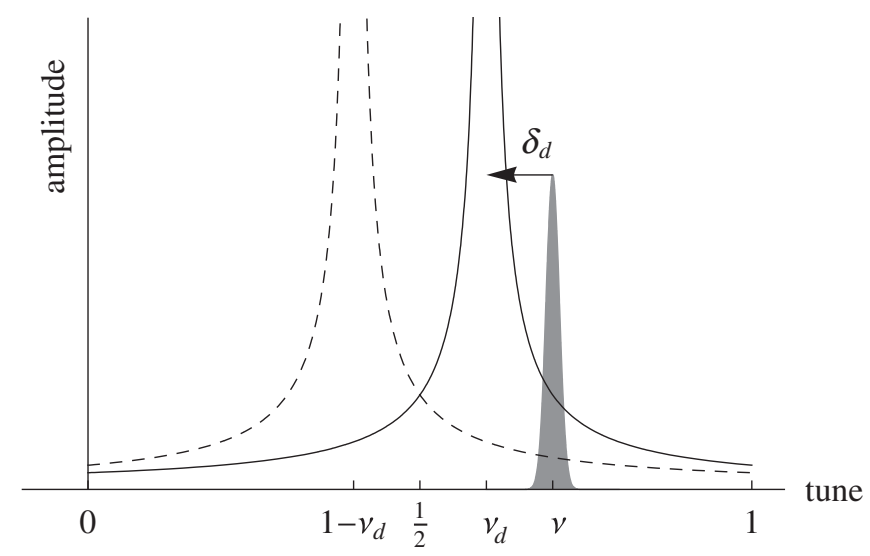

FIG. 2. The amplitudes of the two modes driven by an AC dipole. A circulating beam is driven by the two driving terms of an AC dipole whose driving tunes are $\nu_{d}$ and $1-\nu_{d}$. Solid and dashed curves represent the amplitudes of the two modes excited by these two driving terms for a given machine tune. When the machine tune is $\nu$, beam motion is dominated by the mode of $\nu_{d}$ (solid curve). However, the mode of $1-\nu_{d}$ (dashed curve) cannot always be ignored since realizable $\delta_{d}=\nu_{d}-\nu$ is limited by the tune spread of the beam (shaded area).

It is often convenient to express the longitudinal position $s$ within a circular accelerator using the revolution number $n$, the circumference $C$, and a parameter $\Delta s$ in the range between 0 and $C: s=n C+\Delta s$. Here, the reference point of the longitudinal position $s$ is the location of the AC dipole and the revolution number $n$ increases by one when the beam passes the AC dipole. The transverse position of the beam at $s, x(s)$, can be also written as $x(n C+\Delta s)$. Because of its periodic nature, the $\beta$ function of free oscillations satisfies $\beta(s)=\beta(n C+\Delta s)=\beta(\Delta s)$. The phase advance of free oscillations from $s=0$ to the observation point, $\psi(s)$, satisfies $\psi(s)=\psi(n C+\Delta s)=$ $2 \pi \nu n+\psi(\Delta s)$. As shown in [12,15], when the amplitude of the AC dipole field is constant, the transverse position of the driven beam at the longitudinal position $s=n C+\Delta s$ is given by [16]

$$
\begin{aligned}
x_{d}(n C+\Delta s) \simeq & \frac{\theta_{\text {acd }} \sqrt{\beta_{\text {acd }}}}{4 \sin \left[\pi\left(\nu_{\text {acd }}-\nu\right)\right]} \sqrt{\beta(\Delta s)} \\
& \times \cos \left[2 \pi \nu_{\text {acd }} n+\psi(\Delta s)+\pi\left(\nu_{\text {acd }}-\nu\right)\right. \\
& \left.+\chi_{\text {acd }}\right]+\frac{\theta_{\text {acd }} \sqrt{\beta_{\text {acd }}}}{4 \sin \left[\pi\left(1-\nu_{\text {acd }}-\nu\right)\right]} \\
& \times \sqrt{\beta(\Delta s)} \cos \left[2 \pi\left(1-\nu_{\text {acd }}\right) n+\psi(\Delta s)\right. \\
& \left.+\pi\left(1-\nu_{\text {acd }}-\nu\right)-\chi_{\text {acd }}\right]
\end{aligned}
$$

where $\theta_{\text {acd }}$ is the maximum kick angle of the AC dipole, $\beta_{\text {acd }}$ is the $\beta$ function at the location of the AC dipole, and $\chi_{\text {acd }}$ is the initial phase of the AC dipole field. The reason we express the longitudinal position as $n C+\Delta s$ is because the arguments of the cosines in Eq. (2) undergo discrete 
changes at the location of the AC dipole and cannot be expressed with continuous functions of $s$. The two terms in Eq. (2) are symmetric between $\nu_{\text {acd }}$ and $1-\nu_{\text {acd }}$ and represent the influences of the two driving terms. To quantify the effect of the secondary driving term, it is useful to define a parameter to describe the ratio of the amplitudes of the primary and secondary modes in Eq. (2):

$$
\lambda_{d} \equiv \frac{\sin \left[\pi\left(\nu_{d}-\nu\right)\right]}{\sin \left[\pi\left(1-\nu_{d}-\nu\right)\right]}=\frac{\sin \left(\pi \delta_{d}\right)}{\sin \left(2 \pi \nu+\pi \delta_{d}\right)} .
$$

This parameter $\lambda_{d}$ depends on not only $\delta_{d}$ but also the machine tune $\nu$. When $\left|\delta_{d}\right|=0.01,\left|\lambda_{d}\right| \simeq 0.06$ for the Tevatron $(\nu \simeq 0.58)$ and about half as much for the RHIC and $\operatorname{LHC}(\nu \simeq 0.7$ and 0.3$)$.

\section{B. A new parametrization of driven betatron oscillations}

We note that Eq. (2) can be written in the following compact form which includes the influences of the both driving terms and is expressed with continuous functions of $s$ :

$$
x_{d}(s)=A_{d} \sqrt{\beta_{d}(s)} \cos \left[\psi_{d}(s) \pm \chi_{\text {acd }}\right]
$$

Here, $A_{d}$ is a constant of motion with dimensions (length) $)^{1 / 2}$ :

$$
A_{d} \equiv \frac{\theta_{\text {acd }}}{4 \sin \left(\pi \delta_{d}\right)} \sqrt{\left(1-\lambda_{d}^{2}\right) \beta_{\text {acd }}},
$$

and the sign in front of the phase $\chi_{\text {acd }}$ is positive when $\nu_{d}=\nu_{\text {acd }}$ and negative when $\nu_{d}=1-\nu_{\text {acd }}$. The function $\beta_{d}(s)$ is the newly defined amplitude function of driven oscillations:

$$
\beta_{d}(s) \equiv \frac{1+\lambda_{d}^{2}-2 \lambda_{d} \cos [2 \psi(s)-2 \pi \nu]}{1-\lambda_{d}^{2}} \beta(s)
$$

and $\psi_{d}(s)$ is the newly defined phase advance of driven oscillations from $s=0$ to the observation point:

$$
\psi_{d}(s) \equiv \int_{0}^{s} \frac{d \bar{s}}{\beta_{d}(\bar{s})} .
$$

A relation between the phase advances of free and driven oscillations, $\psi(s)$ and $\psi_{d}(s)$, is given by

$$
\begin{aligned}
\tan \left[\psi_{d}(s)-\pi \nu_{d}\right] & =\frac{1+\lambda_{d}}{1-\lambda_{d}} \tan [\psi(s)-\pi \nu] \\
& =\frac{\tan \left(\pi \nu_{d}\right)}{\tan (\pi \nu)} \tan [\psi(s)-\pi \nu] .
\end{aligned}
$$

When the longitudinal position is given by $s=n C+\Delta s$, $\psi_{d}(s)$ satisfies $\psi_{d}(s)=\psi_{d}(n C+\Delta s)=2 \pi \nu_{d} n+\psi_{d}(\Delta s)$.

In this way, driven betatron oscillations can be parametrized in the same manner as free betatron oscillations when the influences of the two driving terms are included. The difference between driven and free oscillations is
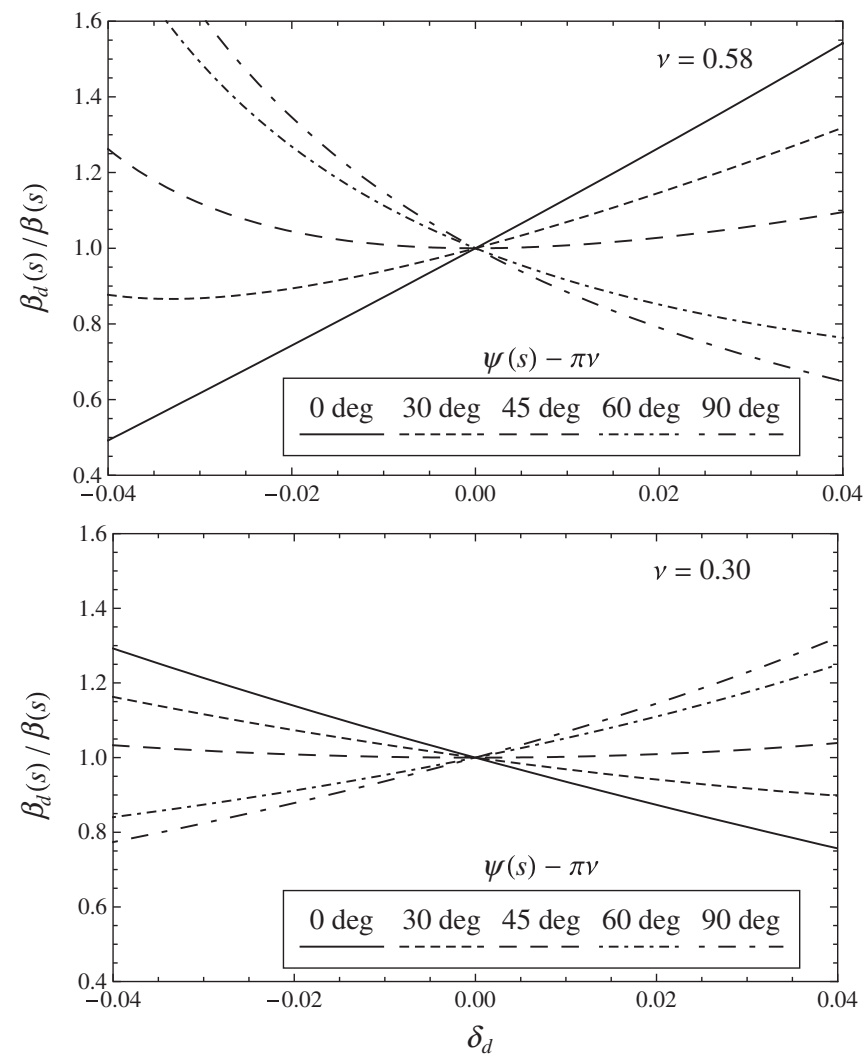

FIG. 3. Ratio between the amplitude functions of driven and free oscillations, $\beta_{d}(s)$ and $\beta(s)$. Based on Eq. (6), $\beta_{d}(s) / \beta(s)$ is calculated for different values of $\delta_{d}=\nu_{d}-\nu$ and $\psi(s)$. When $\nu=0.58$ like the Tevatron, compared to when $\nu=0.3$ like the RHIC and LHC, $\beta_{d}(s) / \beta(s)$ deviates larger from the unity and has stronger nonlinearity because of the larger influence of the secondary driving term.

characterized by the new amplitude function $\beta_{d}(s)$ and phase advance $\psi_{d}(s)$. In the limit of $\nu_{d} \rightarrow \nu, \lambda_{d}$ approaches zero and $\beta_{d}(s)$ and $\psi_{d}(s)$ converge to $\beta(s)$ and $\psi(s)$. Figure 3 shows the numerical calculations of $\beta_{d}(s) / \beta(s)$ based on Eq. (6).

\section{DIFFERENCE BETWEEN FREE AND DRIVEN BETATRON OSCILLATIONS}

Measurement of the $\beta$ function using an $\mathrm{AC}$ dipole requires a careful understanding of the difference between the amplitude functions of free and driven oscillations, $\beta(s)$ and $\beta_{d}(s)$. For free betatron oscillations, the machine tune $\nu$ and amplitude function $\beta(s)$ are correlated, and a change of the tune induces a change of the amplitude function and vice versa. The correlation between the oscillation tune and the amplitude function also applies to driven oscillations. When the beam is driven, the oscillation tune is the primary driving tune $\nu_{d}$ which is different from the machine tune $\nu$. The amplitude function $\beta_{d}(s)$ also differs from $\beta(s)$ for driven oscillations. The relation between $\delta_{d}=\nu_{d}-\nu$ and $\beta_{d}(s) / \beta(s)$ is formally the same 
as the tune shift and the change of the $\beta$ function produced by a thin gradient error. This analogy helps to interpret data of driven oscillations.

\section{A. Review of a thin gradient error}

When an accelerator has a gradient error, its machine tune and $\beta$ function change [1]. We assume there is a thin gradient error with gradient $B_{1}$ and length $\ell$ at a longitudinal position $\Delta s=0$ within a synchrotron and the gradient error changes the machine tune and the amplitude function to $\nu_{q}$ and $\beta_{q}(s)$. Then, the equation of motion is given by

$$
x^{\prime \prime}(s)+K(s) x(s)=-q_{\mathrm{err}}\left[\sum_{n=-\infty}^{\infty} \delta(s-C n)\right] x(s) .
$$

Here, the prime denotes the derivative with respect to the longitudinal position $s, K(s)$ is the effective focusing function, $q_{\mathrm{err}}=B_{1} \ell /(B \rho)$ is the effective strength of the gradient error, $(B \rho)$ is the magnetic rigidity, and $\delta(s)$ is the Dirac delta function. As in the previous section, $n$ is the revolution number and we choose the location of the gradient error as the reference point of the longitudinal position $s$.

By comparing the single turn transfer matrices with and without the gradient error, we get the following two equations for $\nu_{q}$ and $\beta_{q}(s)$ :

$$
\begin{gathered}
q_{\mathrm{err}}=2 \frac{\cos (2 \pi \nu)-\cos \left(2 \pi \nu_{q}\right)}{\beta_{\mathrm{err}} \sin (2 \pi \nu)} \\
\beta_{q}(s)=\frac{\sin (2 \pi \nu)-q_{\mathrm{err}} \beta_{\mathrm{err}} \sin (\psi(s)) \sin [2 \pi \nu-\psi(s)]}{\sin \left(2 \pi \nu_{q}\right)} \beta(s),
\end{gathered}
$$

where $\beta_{\text {err }}$ is the $\beta$ function at the location of the gradient error, $\delta_{q} \equiv \nu_{q}-\nu$ is the tune shift caused by the gradient error. As in the previous section, $\psi(s)$ is the phase advance of free oscillations from $s=0$ to the observation point when the accelerator does not have this gradient error. By substituting the first equation into the second, $\beta_{q}(s)$ is given by

$$
\beta_{q}(s)=\frac{1+\lambda_{q}^{2}-2 \lambda_{q} \cos [2 \psi(s)-2 \pi \nu]}{1-\lambda_{q}^{2}} \beta(s) .
$$

Here, $\lambda_{q}$ is defined as a parameter with a similar form to $\lambda_{d}:$

$$
\lambda_{q} \equiv \frac{\sin \left(\pi \delta_{q}\right)}{\sin \left(2 \pi \nu+\pi \delta_{q}\right)} .
$$

When $\lambda_{q}$ is small, the new and original amplitude functions satisfy

$$
\frac{\beta_{q}(s)-\beta(s)}{\beta(s)} \simeq-2 \lambda_{q} \cos [2 \psi(s)-2 \pi \nu] .
$$

This quantity behaves like a standing wave in the synchrotron and is called $\beta$ beat or $\beta$ wave. The amplitude of the $\beta$ beat is $2\left|\lambda_{q}\right|$ at lowest order.

It may be seen from Eqs. (6) and (12) that the relation between $\beta_{d}(s)$ and $\delta_{d}$ for driven oscillations is similar to the relation between $\beta_{q}(s)$ and $\delta_{q}$ when there is a thin gradient error. Hence, relative to $\beta(s)$, we expect $\beta_{d}(s)$ will beat with amplitude of $2\left|\lambda_{d}\right|$.

\section{B. Analogy between an AC dipole and gradient error}

This section explains why an oscillating dipole field changes the observed phase space motion, much like a gradient error. When driven by the $\mathrm{AC}$ dipole, the equation of motion is given by

$$
x^{\prime \prime}(s)+K(s) x(s)=-\theta_{\text {acd }} \sum_{n} \cos \left(2 \pi \nu_{d} n \pm \chi_{\text {acd }}\right) \delta(s-C n) .
$$

The right-hand side describes the kicks by the AC dipole located at $\Delta s=0$. The summation of the revolution number $n$ runs over the time period when the amplitude of the $\mathrm{AC}$ dipole field is constant and the sign in front of the initial phase $\chi_{\text {acd }}$ follows the same convention as Eq. (4). Equation (4) is the particular solution of this inhomogeneous Hill's equation when the amplitude of the AC dipole field is adiabatically ramped up to a constant amplitude. From Eq. (4), the transverse position of the driven beam at the location of the AC dipole, $\Delta s=0$, is given by

$$
x_{d}(C n)=A_{d} \sqrt{\beta_{d}(0)} \cos \left(2 \pi \nu_{d} n \pm \chi_{\mathrm{acd}}\right) .
$$

We note that the phases in Eqs. (15) and (16) are the same. Hence, the AC dipole field is in sync with the transverse position of driven oscillations when the beam passes the AC dipole. The situation is analogous to a quadrupole magnet whose field is proportional to a transverse position.

Because $x_{d}(s)$ is the solution of Eq. (15), it formally satisfies the following equation:

$$
x_{d}^{\prime \prime}(s)+K(s) x_{d}(s)=-q_{\text {acd }}\left[\sum_{n} \delta(s-C n)\right] x_{d}(s) .
$$

Here, Eq. (16) is used to rewrite $\cos \left(2 \pi \nu_{d} n \pm \chi_{\text {acd }}\right)$ with $x_{d}(s)$. The parameter $q_{\text {acd }}$ is a constant analogous to the effective strength of a gradient error $q_{\text {err }}$ :

$$
q_{\mathrm{acd}} \equiv \frac{\theta_{\mathrm{acd}}}{A_{d} \sqrt{\beta_{d}(0)}}=2 \frac{\cos (2 \pi \nu)-\cos \left(2 \pi \nu_{d}\right)}{\beta_{\mathrm{acd}} \sin (2 \pi \nu)} .
$$

Equation (17) has exactly the same form as the equation of motion when there is a thin gradient error, Eq. (9). By comparing Eqs. (9), (10), (17), and (18), it is trivial that the relation between $\beta_{d}(s)$ and $\delta_{d}$ is the same as the relation between $\beta_{q}(s)$ and $\delta_{q}$. 


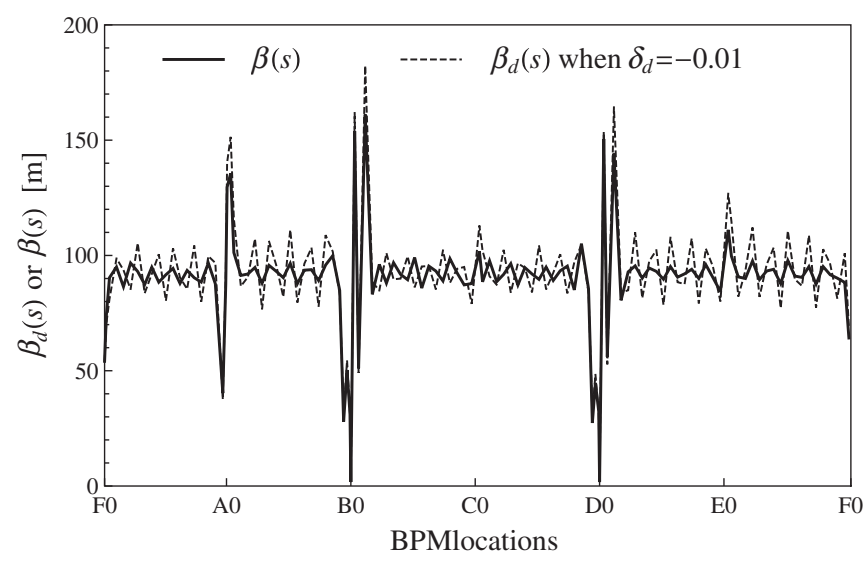

FIG. 4. The amplitude functions of free and driven oscillations, $\beta(s)$ (solid) and $\beta_{d}(s)$ when $\delta_{d}=-0.01$ (dashed), in the Tevatron. As expected, $\beta_{d}(s)$ shows beating with $10 \%-15 \%$ peak height relative to $\beta(s)$. From multiple data sets of driven oscillations, the amplitude function of free oscillations, $\beta(s)$, can be extrapolated.

\section{Ring-wide behavior of the amplitude function $\boldsymbol{\beta}_{d}(s)$}

When turn-by-turn beam positions are recorded by BPM's for free oscillations, the relative $\beta$ function can be determined by simply comparing the square of the oscillation amplitudes. If the same analysis is applied to turn-by-turn data of driven oscillations, what is calculated is $\beta_{d}(s)$ instead of $\beta(s)$. From the analogy between an AC dipole and a gradient error, the normalized difference between $\beta_{d}(s)$ and $\beta(s)$ changes sinusoidally with BPM location:

$$
\frac{\beta_{d}(s)-\beta(s)}{\beta(s)} \simeq-2 \lambda_{d} \cos [2 \psi(s)-2 \pi \nu] .
$$

This beating of $\beta_{d}(s)$ relative to $\beta(s)$ cannot be distinguished from the $\beta$ beat caused by gradient errors. When the magnitude of the parameter $\delta_{d}$ is 0.01 , the peak value of the beating $2\left|\lambda_{d}\right|$ is about $12 \%$ for the Tevatron and $6 \%$ for the RHIC and LHC [17]. Figure 4 shows $\beta(s)$ and $\beta_{d}(s)$ in the case of $\delta_{d}=-0.01$ in the Tevatron.

As explained in the following section, by using multiple data sets of driven oscillations, the influences of the primary and secondary driving terms can be separated and the $\beta$ function of free oscillations can be measured without depending on a machine model.

\section{EFFECT ON $\beta$-FUNCTION MEASUREMENT}

\section{A. Rotation of the phase space ellipse}

The previous section discussed the amplitude function of driven oscillations $\beta_{d}(s)$. Parameters corresponding to the other Courant-Snyder parameters $\alpha(s)$ and $\gamma(s)$ [1] can be also defined for driven oscillations:

$$
\alpha_{d}(s) \equiv-\frac{1}{2} \frac{d \beta_{d}(s)}{d s}
$$

$$
\gamma_{d}(s) \equiv \frac{1+\alpha_{d}(s)^{2}}{\beta_{d}(s)}
$$

The explicit forms of these parameters are given by

$$
\begin{aligned}
\alpha_{d}(s)= & \frac{1+\lambda_{d}^{2}-2 \lambda_{d} \cos [2 \psi(s)-2 \pi \nu]}{1-\lambda_{d}^{2}} \alpha(s) \\
& -\frac{2 \lambda_{d} \sin [2 \psi(s)-2 \pi \nu]}{1-\lambda_{d}^{2}}
\end{aligned}
$$

and

$$
\begin{aligned}
\gamma_{d}(s)= & \frac{1+\lambda_{d}^{2}+2 \lambda_{d} \cos \{2 \psi(s)-2 \pi \nu+2 \arctan [\alpha(s)]\}}{1-\lambda_{d}^{2}} \\
& \times \gamma(s) .
\end{aligned}
$$

When $\beta_{d}(s), \alpha_{d}(s), \gamma_{d}(s)$, and $A_{d}$ are defined in this way, they satisfy the Courant-Snyder invariance:

$$
A_{d}^{2}=\gamma_{d}(s) x_{d}(s)^{2}+2 \alpha_{d}(s) x_{d}(s) x_{d}^{\prime}(s)+\beta_{d}(s) x_{d}^{\prime}(s)^{2} .
$$

Hence, the turn-by-turn position and angle of driven oscillations form an ellipse in phase space, just like free oscillations. The Courant-Snyder-like parameters $\beta_{d}(s), \alpha_{d}(s)$, and $\gamma_{d}(s)$ depend on $\delta_{d}$, so the area and shape of the phase space ellipse changes with $\delta_{d}$ for driven oscillations. Because $\beta_{d}(s), \alpha_{d}(s)$, and $\gamma_{d}(s)$ converge into $\beta(s)$, $\alpha(s)$, and $\gamma(s)$ in the limit of $\lambda_{d} \rightarrow 0$, this change of the shape is due to the secondary driving term.

In two collision straight sections of the Tevatron there are pairs of BPM's with no magnetic element in-between. The beam travels along straight lines between these pairs and, hence, both position and angle can be directly measured at these locations. Figure 5 shows the phase space ellipses of driven oscillations measured with a pair of BPM's at an interaction point. In these measurements, $\delta_{d}$

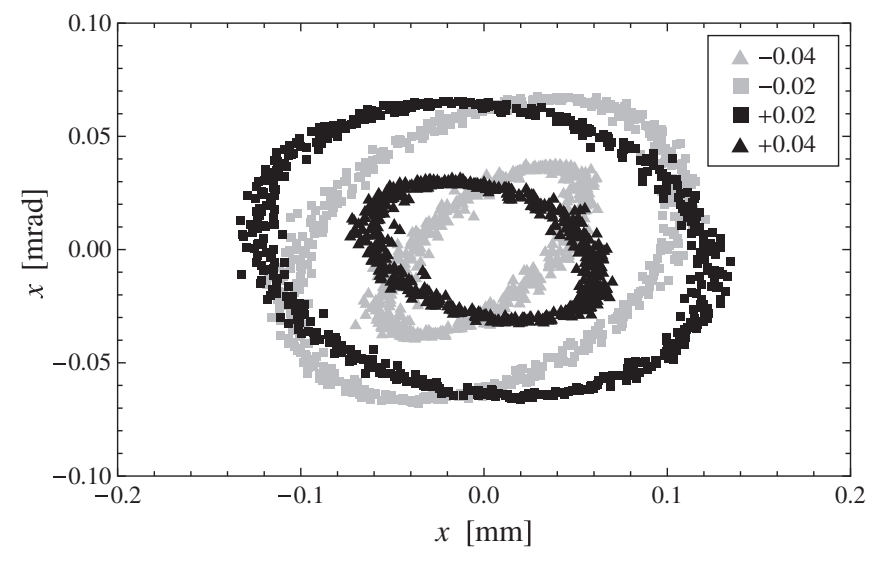

FIG. 5. Phase space ellipses of driven oscillations at an interaction point in the Tevatron (B0) when $\delta_{d}= \pm 0.02$ and \pm 0.04 . Because the Courant-Snyder-like parameters of driven oscillations $\beta_{d}(s), \alpha_{d}(s)$, and $\gamma_{d}(s)$ depend on $\delta_{d}$, not only the areas but also the shapes of the ellipses are different. 


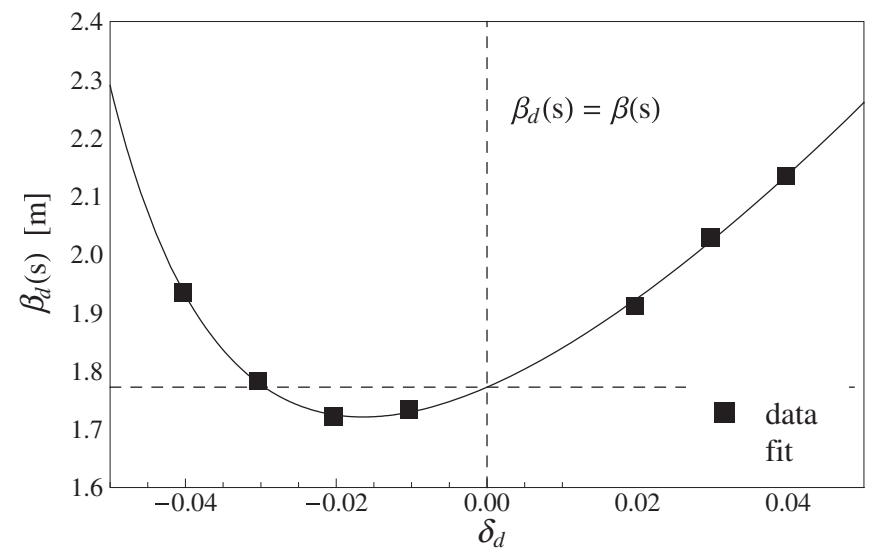

FIG. 6. $\beta_{d}(s)$ vs $\delta_{d}$ at an interaction of the Tevatron (B0). Each data point is from the fit of Eq. (24) to an ellipse in Fig. 5. The curve is the fit of Eq. (6) to the data points. The true $\beta$ function of this location is the value of $\beta_{d}(s)$ when $\delta_{d}=0$.

is set to \pm 0.04 and \pm 0.02 , while the kick angle of the $\mathrm{AC}$ dipole $\theta_{\text {acd }}$ is kept the same. As expected, the shape of the ellipse changes with $\delta_{d}$.

By fitting Eq. (24) to an ellipse in Fig. 5, its area $\pi A_{d}^{2}$ and the parameters $\beta_{d}(s), \alpha_{d}(s)$, and $\gamma_{d}(s)$ can be determined. Figure 6 shows $\beta_{d}(s)$ determined from the fits to the ellipses in Fig. 5 (and three more). The curve in the figure is the fit of Eq. (6) to the data with parameters $\beta(s)$ and $\psi(s)$. We can see the model of Eq. (6) fits well to the data. In the figure, the true $\beta$ function at this location is obtained by extrapolation of $\beta_{d}(s)$ to the case of $\delta_{d}=0$. In the Tevatron, the $\beta$ function at the two interaction points can be measured in this way.

\section{B. Asymmetric amplitude response}

When the influence of the secondary driving term is negligible, by ignoring the smaller term of Eq. (2) or taking the limit of $\lambda_{d} \rightarrow 0$ in Eqs. (5) and (6), the amplitude of driven oscillations is approximated by

$$
a_{d}^{(0)}(s) \equiv\left[\left|A_{d}\right| \sqrt{\beta_{d}(s)}\right]_{\lambda_{d} \rightarrow 0}=\frac{\theta_{\mathrm{acd}} \sqrt{\beta_{\mathrm{acd}} \beta(s)}}{4 \sin \left(\pi\left|\nu_{d}-\nu\right|\right)} .
$$

In this limit, the amplitude depends on the primary driving tune $\nu_{d}$ only through $\sin \left(\pi\left|\nu_{d}-\nu\right|\right)$ and is symmetric around the machine tune $\nu$. From Eqs. (5) and (6), the amplitude including the influence of the secondary driving term $a_{d}(s)$ is given by

$$
\begin{aligned}
a_{d}(s) & \equiv\left|A_{d}\right| \sqrt{\beta_{d}(s)} \\
& =a_{d}^{(0)}(s) \sqrt{1+\lambda_{d}^{2}-2 \lambda_{d} \cos [2 \psi(s)-2 \pi \nu]} .
\end{aligned}
$$

Here, $a_{d}(s)$ depends on $\nu_{d}$ through the factor $\left\{1+\lambda_{d}^{2}-\right.$ $\left.2 \lambda_{d} \cos [2 \psi(s)-2 \pi \nu]\right\}^{1 / 2}$ as well. To the first order of $\nu_{d}-\nu$,

$$
a_{d}(s) \simeq a_{d}^{(0)}(s)\left[1-\frac{\pi \cos [2 \psi(s)-2 \pi \nu]}{\sin (2 \pi \nu)}\left(\nu_{d}-\nu\right)\right]
$$

Hence, the secondary driving term makes the $\nu_{d}$ dependence of the amplitude asymmetric around the machine tune $\nu$. Because of the factor $\cos [2 \psi(s)-2 \pi \nu]$, the magnitude of this asymmetry depends on a location in a synchrotron.

Figure 7 shows the relation between the amplitude of driven oscillations and $\nu_{d}$ at three BPM locations in
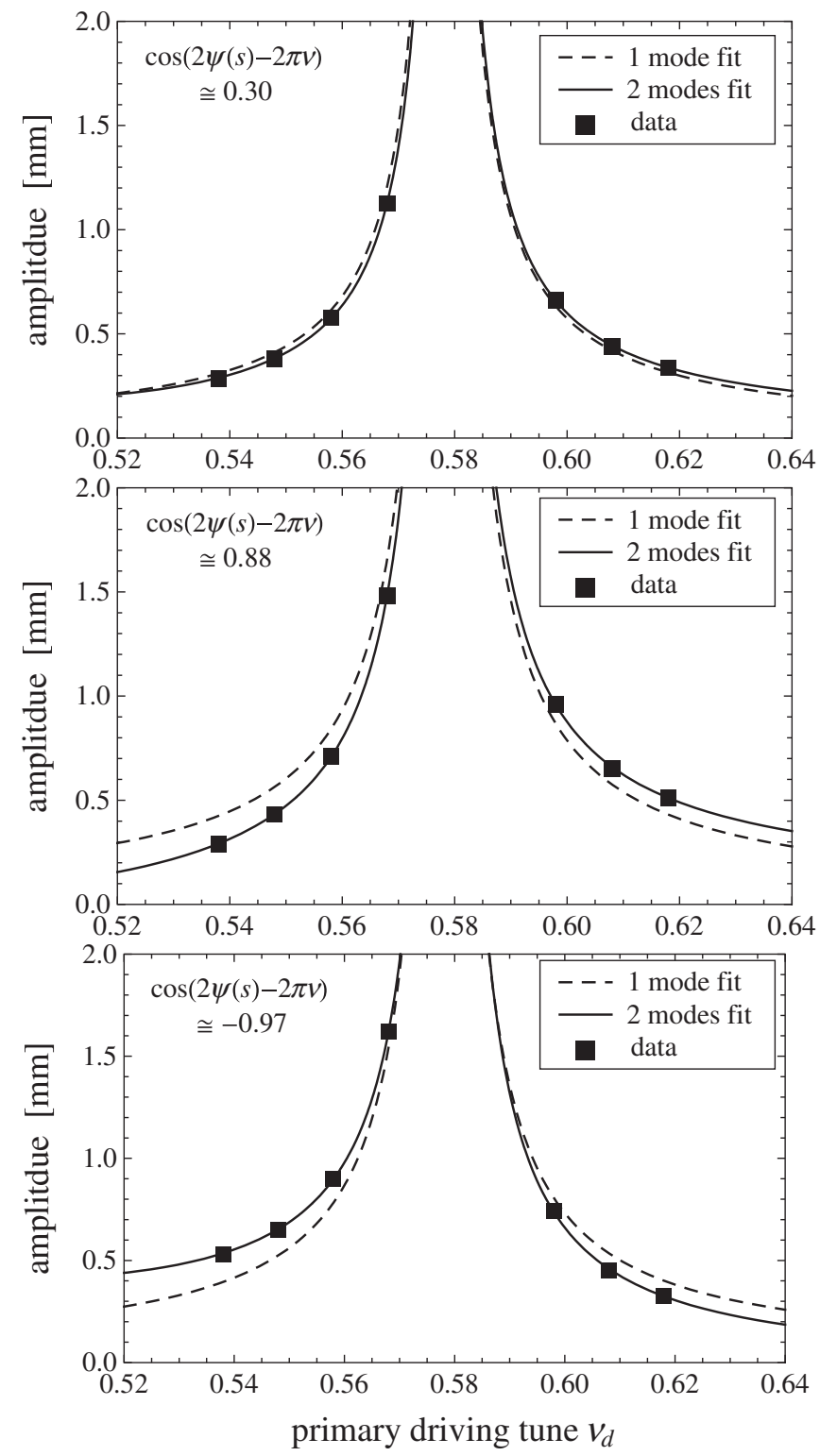

FIG. 7. The amplitude of driven oscillations vs the primary driving tune $\nu_{d}$ at three BPM locations in the Tevatron. Two curves represent the fits when the secondary driving term is included (solid) and not included (dashed). The asymmetries around the machine tune $\nu \simeq 0.5785$ depend on the factor $\cos [2 \psi(s)-2 \pi \nu]$. 


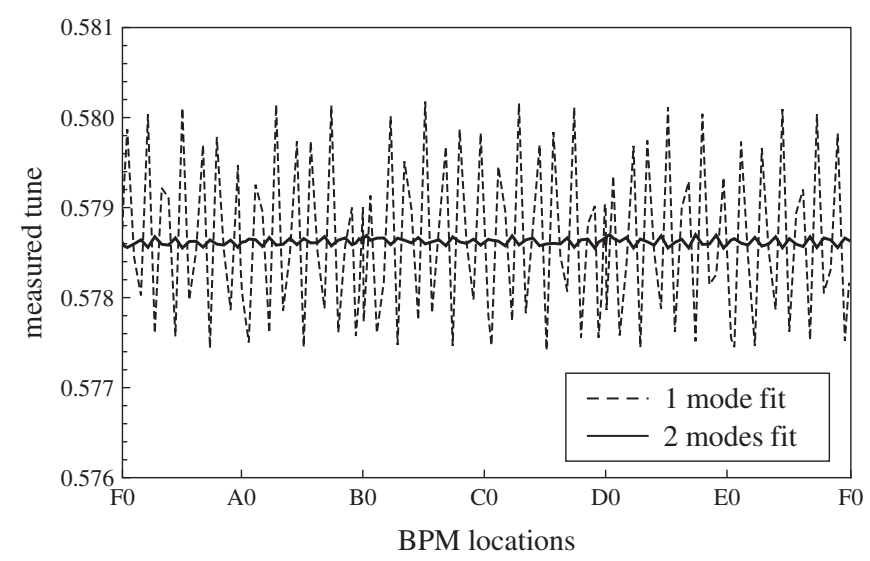

FIG. 8. The machine tune derived from the fit of the amplitude response at each BPM location. Inclusion of the secondary driving term gives a much more consistent result for the machine tune (global parameter).

the Tevatron. The dashed and solid curves represent the fits of Eqs. (25) and (26) to the data. The fit parameters are $\theta_{\text {acd }}\left[\beta_{\text {acd }} \beta(s)\right]^{1 / 2}$ and $\nu$ for Eq. (25) and $\theta_{\text {acd }}\left[\beta_{\text {acd }} \beta(s)\right]^{1 / 2}$, $\nu$, and $\psi(s)$ for Eq. (26). At two locations where $|\cos [2 \psi(s)-2 \pi \nu]|$ is close to one, the asymmetries around the machine tune $(\nu \simeq 0.5785)$ are large and the fits of Eq. (25), which ignores the secondary driving term, is not well matched. From the fit of Eq. (26), the $\beta$ function at each BPM location is determined up to a constant $\theta_{\text {acd }}\left(\beta_{\text {acd }}\right)^{1 / 2}$. This constant can be determined from the analysis in the previous section which uses a pair of BPM's in a collision straight section. By combining these two types of analyses, the ring-wide $\beta$ function can be directly measured from multiple data sets of driven oscillations with different $\nu_{d}$.

From the fits in Fig. 7, the machine tune $\nu$ is determined at each BPM location. Figure 8 shows the machine tunes determined from the fits at all the BPM locations. The solid curve includes the influence of the secondary driving term and the dashed curve does not. Because the machine tune $\nu$ is a global parameter of an accelerator, the model including the two modes works better. The residual variation of the determined tunes in the analysis of the two modes model can be contributed by BPM noises and other systematic effects.

\section{CONCLUSION}

Under the influence of a sinusoidally oscillating magnetic field of an AC dipole, the beam is driven by two driving terms. As a result, the phase space trajectory of driven betatron oscillations is different from that of free betatron oscillations. If this difference is simply ignored, interpretations of the linear optical parameters based on data of driven oscillations can have errors depending on the driving tune and the machine tune. In this paper, we show that this change of the phase space trajectory is formally identical to the change induced by a gradient error at the same location as the AC dipole. Just as a gradient error changes the amplitude function and phase advance, the expression of driven oscillations can be simplified by introducing a new amplitude function and phase advance.

This paper presents a few examples of the difference between free and driven oscillations as observed in the Tevatron. It also shows that the new parametrization of driven oscillations clarifies the interpretation of turn-byturn data of driven oscillations.

With this knowledge, very precise and direct measurements of the true linear optical parameters in a synchrotron can be obtained quickly without degradation of the beam quality, using a small number of data sets obtained at different frequencies of the $\mathrm{AC}$ dipole. This technique will be especially useful in the LHC, for example, to adjust the beam envelope at critical locations such as at beam collimation devices.

[1] Betatron oscillation is discussed in standard textbooks of accelerator physics such as by D. A. Edwards and M. J. Syphers [An Introduction to the Physics of High Energy Accelerators (John Wiley \& Sons, Inc., New York, 1993)] or by S. Y. Lee [Accelerator Physics (World Scientific, Singapore, 2004), 2nd ed.].

[2] We use machine tune for the tune of free betatron oscillations to distinguish it from the tune of driven betatron oscillation, which is defined later.

[3] M. Bai, S. Y. Lee, J. W. Glenn, H. Huang, L. Ratner, T. Roser, M. J. Syphers, and W. van Asselt, Phys. Rev. E 56, 6002 (1997).

[4] M. Bai, M. Meth, C. Pai, B. Parker, S. Peggs, T. Roser, R. Sanders, D. Trbojevic, and A. Zaltsman, in Proceedings of the 19th Particle Accelerator Conference, Chicago, Illinois, 2001 (IEEE, Piscataway, NJ, 2001), p. 3606.

[5] M. Bai, J. Delong, L. Hoff, C. Pai, S. Peggs, J. Piacentino, B. Oerter, P. Oddo, T. Roser, T. Satogata, D. Trbojevic, and A. Zaltsman, in Proceedings of the 8th European Particle Accelerator Conference, Paris, France, 2002 (EPS-IGA and CERN, Geneva, 2002), p. 1115.

[6] O. Berrig, W. Hofle, R. Jones, J. Koopman, J. P. Koutchouk, H. Schmickler, and F. Schmidt, in Proceedings of the 5th European Workshop on Beam Diagnostics and Instrumentation for Particle Accelerators, Grenoble, France, 2001 (ESRF, Grenoble, 2001), p. 82.

[7] F. Schmidt, R. Tomás, N. Catalán, G. Crockford, M. Hayes, W. Hofle, and J.P. Koutchouk, CERN Report No. AB-Note-2003-031 MD, 2003.

[8] R. Miyamoto, A. Jansson, S. Kopp, and M. Syphers, in Proceedings of the 12th Beam Instrumentation Workshop, Batavia, Illinois, 2006 (AIP, Melville, New York, 2006), p. 402.

[9] R. Miyamoto, S. E. Kopp, A. Jansson, and M. J. Syphers, in Proceedings of the 22nd Particle Accelerator Conference, Albuquerque, New Mexico, 2007 (IEEE, Piscataway, NJ, 2007). 
[10] R. Miyamoto, S. E. Kopp, A. Jansson, and M. J. Syphers, in Proceedings of the 22nd Particle Accelerator Conference, Albuquerque, New Mexico, 2007 (Ref. [9]).

[11] M. Bai, R. Calaga, W. Fischer, P. Oddo, H. Schmickler, J. Serrano, A. Jansson, M. J. Syphers, S. Kopp, and R. Miyamoto, in Proceedings of the 22nd Particle Accelerator Conference, Albuquerque, New Mexico, 2007 (Ref. [9]) and arXiv:0709.2753.

[12] S. Peggs, in Proceedings of the 18th Particle Accelerator Conference, New York, New York, 1999 (IEEE, Piscataway, NJ, 1999), p. 1572.

[13] This analogy is based on properties of the amplitude function of driven oscillations $\beta_{d}(s)$ which is defined in Sec. II. As the $\beta$ function of free oscillations is well defined only in the linear regime [1], our analogy applies only to the linear regime.

[14] This is a point of view from the circulating beam. There is only one betatron tune $\nu$ for the circulating beam but it cannot tell whether the AC dipole tune is $\nu_{\text {acd }}$ or $1-\nu_{\text {acd }}$, and the beam is driven by both of these driving tunes. On the contrary, from a point of view of the AC dipole, there is only one AC dipole tune $\nu_{\text {acd }}$ but it cannot tell whether the beam's betatron tune is $\nu$ or $1-\nu$, and it drives both of these modes. Although the second picture may be easier to understand for an accelerator physicist, we use the first picture in this paper since it is convenient when we solve the equation of motion.

[15] R. Tomás, Phys. Rev. ST Accel. Beams 8, 024401 (2005).

[16] Equation (2) is assuming the amplitude of the AC dipole field is adiabatically ramped up to a constant value. The exact expression of $x_{d}(s)$, which is given in [15], includes transient modes which are inversely proportional to the ramp-up time and oscillate with the machine tune $\nu$. If the ramp-up is slow enough, all the transient modes are very small and decohere before the end of the ramp-up. Hence, these ignored modes do not affect motion of the beam centroid.

[17] We note that a possible range of the parameter $\delta_{d}$ depends on an accelerator and its condition. Here, $\left|\delta_{d}\right|=0.01$ is chosen for the estimations because it is a typical limit of the Tevatron and also easy to be scaled. At the injection energy of the Tevatron, $\left|\delta_{d}\right|=0.01$ is sometimes too small and the beam is often driven with $\left|\delta_{d}\right|=0.015$. In this case, the normalized difference $\left[\beta_{d}(s)-\beta(s)\right] / \beta(s)$ increases to about $18 \%$. On the contrary, if the beam is driven with smaller $\left|\delta_{d}\right|$ than 0.01 as achieved in the AGS, SPS, and Tevatron $[3,6,7,18]$, the normalized difference between $\beta_{d}(s)$ and $\beta(s)$ becomes smaller than $12 \%$ and $6 \%$ for each.

[18] C. Y. Tan and V. H. Ranjbar, Phys. Rev. ST Accel. Beams 11, 032802 (2008). 